\title{
The Pharmacological Action of Common Herbal Remedies
}

\author{
Rashid Bhikha*, John Glynn \\ Email address: \\ rashidb@tibb.co.za (R. Bhikha),jpglynn@mweb.co.za (J. Glynn) \\ ${ }^{*}$ Corresponding author
}

Department of Research, The Ibn Sina Institute of Tibb, Johannesburg, South Africa

\section{To cite this article:}

Rashid Bhikha, John Glynn. The Pharmacological Action of Common Herbal Remedies. American Journal of Internal Medicine. Vol. 6, No. 5, 2018, pp. 99-107. doi: 10.11648/j.ajim.20180605.13

Received: July 2, 2018; Accepted: July 27, 2018; Published: August 24, 2018

\begin{abstract}
Acceptance of the clinical efficacy and tolerance of herbal remedies by practitioners of conventional, Western medicine is strongly dependent on unambiguous and reproducible evidence, particularly their pharmacological action. A single conventional drug generally has one dominant pharmacological mode of action which accounts for its therapeutic efficacy, whereas a herbal remedy because of the various active agents it contains, generally influences several biochemical and/or physiological systems present in the body. Identifying this therefore poses several challenges. They are much more complex, and the active components of a herbal remedy may act synergistically or interact in other ways. Although the need to determine modes of action has not been a primary concern of complementary healthcare practitioners, there is increasing pressure to elaborate objectively on their pharmacological action. In response, there has been an encouraging, and escalating, release of scientific data documenting the mode of action of many herbal remedies. Some we know about in considerable detail; of others we have an inkling; but for the vast majority the' modes of action remain to be elucidated. This brief review, based on a survey of the available scientific and clinical literature, examines the modes of action of several therapeutic herbs which are being increasingly used, whether informally or as part of complementary or integrative medical practice. It examines a major category of sub stances, the adaptogens, and the various chemical forms it embraces, such as the saponins and triterpenes. The herbs with modulate the immune system, for instance garlic, turmeric and St John's Wort, are likewise surveyed, as are the alkaloids and botanical anti-inflammatory agents. Herbs which exert pharmacological action via their nitric oxide regulatory properties are listed, as are those which provide essential micro-nutrients, such as vitamins and certain minerals. The review concludes with comment on the importance of understanding herbal remedy modes of action as a positive step to their wider adoption in the modern-day therapeutic armamentarium.
\end{abstract}

Keywords: Herbs, Pharmacology, Adaptogens, Alkaloids, Immune Modulators, Anti-inflammatories, NO Synthesis Inhibitors, Micronutrients

\section{Introduction}

Identifying the pharmaceutical mechanisms of herbal products poses numerous challenges not faced by conventional drugs. [1,2] For example, they contain many active substances which often interact in combination or synergistically, whereas conventional drugs are generally studied in isolation as single agents. [3, 4] Another difference is that a single conventional drug may have only one major direct action, but the herbal product will probably have several. What's more, although the herbal product may contain a particular active agent, it may not be present in sufficient quantity to elicit a pharmacological action. Yet another difference is that complex pharmacological synergy may operate within a herbal remedy; that is, the net pharmacological response will be different when compared to one active agent alone, as the dominant mechanism may be potentiated by other, separate and variable influences.

There is a powerful body of opinion that feels, or even insists, that clinical evidence of efficacy and tolerance for the myriad of herbal products presently on the market should be provided, and examined critically. [5-7] Unfortunately, there is one major problem with conducting research into herbal products: there is a much higher degree of complexity in herbal remedies when compared to conventional drugs. In fact, there may be hundreds of different activities present in a 
single herb, and many herbal remedies combine several herbs. Herbal medicines, therefore, generally have to be assessed on traditional and empirical experience. [8, 9, 42]

An important and highly relevant factor is that producers of herbal medicines have little or no access to the massive funding available to the research institutions and "Big Pharma" to carry out large, well-controlled and planned clinical studies. A small, double-blind, cross-over clinical trial costs several million dollars to organise, manage, control, analyse, and report. As a result, information on the modes of action of herbal remedies is accumulating, but at a relatively slow pace. Even so, much is known about the pharmacology of certain popular herbs, especially those used in the industrialised countries, partly because they have been around for a long time $[9,10]$.

\section{Herb Pharmacology}

Compared to the mechanisms of action of herbal products, conventional pharmacology is relatively straightforward. [3, 4, 11, 38]. Drugs exert their effects for the most part in a discrete number of ways. They can stimulate, or inhibit, or otherwise interfere with, the functioning of neurotransmitters and drug receptors, they can reduce the activity of certain enzymes involved in key biochemical or metabolic processes, or they can affect cell membrane channels and permeability. In contrast, herbs exert their action in a variety of ways: some we know about in considerable detail; for others we have an inkling of their modes of action; but for many, the modes of action still remain to be elucidated. [12]

Although the therapeutic effects of many herbal remedies are well documented, details of their actual pharmacological actions have not yet been clearly described. There are several reasons for this. Firstly, herbal products have been around for millennia, and the efficacy of specific traditional remedies has been established empirically, by extensive clinical experience and observation. Examples are ginger, turmeric and garlic. [43] Herbal remedies that have been proven effective since time immemorial, such as these, have retained their place in healthcare practice, whereas those that failed to deliver expected treatment outcomes have fallen by the wayside. Therefore, the need to determine the mechanisms of action has not been, from the historical perspective, a primary concern to complementary healthcare practitioners.

Another important factor is the financial largesse available to researchers into conventional drugs Research \& Development in general, and their pharmacological natures in particular. This is generously provided by the transnational and local pharmaceutical companies, and supported by government departments, private institutes and medical schools. This funding is rarely accessible by herbal remedy providers or practitioners, and even only grudgingly to researchers.

An important feature of herbal medication is that it acts holistically. A herb contains many active and chemically varied components and secondary metabolites. These often influence several biochemical and/or physiological system present in the living body. [9, 13] In addition, there may be marked effects at different existential levels - the subcellular, biochemical, humoral, even metaphysical. As such, the positive impact of an herb on the person's intrinsic or innate self-healing mechanisms - physis [14] - contributes to the resultant clinical improvement.

\section{Specific Mechanisms of Action of Herbal Remedies}

\subsection{Adaptogens}

An adaptogen is a plant-derived substance which acts to increase a person's resistance to stress, trauma, anxiety, and fatigue. [15] When used by herbalists, the term refers to herbs which are more popularly known as tonics, agents which have a rejuvenating effect. [16] It includes Q tonics in Chinese herbal medicine, rasayanas in Ayurvedic medicine, and multi-herb restoratives in Tibb medicine. Adaptogen-rich plants such as licorice and ginseng have a long history of use as tonics in traditional healing practices. [17]

There are no equivalent drugs in conventional medicine, although synthetic vitamins and supplements are often promoted to boost the metabolism of both healthy and sick people, and those who are pregnant, nursing, or convalescing.

As with tonics in general, the effect is most pronounced in those people showing signs and symptoms of a disturbed internal harmony, or homeostasis, and these are the ones who stand to benefit. People who are not in need of a tonic may perceive improved energy and vitality, but many consider this due to psychological upliftment or the placebo response.

A separate group of herbal products with adaptogenic action, the immune-modulators (see later), have the ability to stimulate the immune system in order to deal with infection and cancer, while at the same time decreasing the immune processes that promote inflammation in wounds and other injuries.

\subsubsection{Mechanism of Action}

Adaptogens are able to speed up or slow down a number of body functions, depending on the needs at the time. They act as metabolic checks and balances, and help maintain or restore the body's homeostatic status. [18] They achieve this by influencing both the immune system and the endocrine system, so restoring harmony to the body when disturbed by certain disorders. The active ingredients in the herbs, once absorbed into the body, are able to penetrate the cell membrane. Some are capable of moving, and then attaching, to the DNA of the nucleus. This interaction leads to modified secretion of hormones and transfer of messages by the nervous system. In addition, secretion of hormones from the endocrine system is regulated. A number of adaptogens are structurally made up of polysaccharides. Others are plant sterols (phytosterols), which are based on sitosterol, a steroid analogous to cholesterol in humans. Adaptogens as a class appear to act as anti-oxidants, so limiting the impact of free radicals and oxidative species on the body's metabolic 
processes. [19]

\subsubsection{Desired Clinical Effects}

The beneficial effects of the adaptogens, confirmed empirically over the years, are many and varied. Their effect on the body is to help in coping with metabolic stress, so limiting the disturbance of internal homeostasis. [20] They improve the body's immune competence and resistance, so protecting against the deleterious effects of pathogens. In addition, they support the liver, so helping to oppose toxic influences from the diet or the environment. They improve blood sugar metabolism, resulting in alleviation of fatigue and provide energy at critical times during, for example, infection and convalescence. They help to accelerate recovery from convalescence, or from the trauma of injury or surgery. Moreover, adherents of the adaptogens claim they improve muscle tone when needed. Psychologically, they seem to improve a person's focus and concentration, decrease anxiety, and correct disturbed sleeping patterns.

\subsubsection{Adaptogens in Practice}

An example of an adaptogen in action is evident in hawthorn berries. These have been used to normalise blood pressure: if too high, the adaptogen will reduce it, if too low, it will raise it. Another example concerns the use of valerian in anxiety. One herbal component elicits a sedative action, whereas another will have a slight stimulatory effect. The net result is a dynamic, well-controlled anxiety-lowering effect, without unwelcome side effects such as sluggishness.

\subsubsection{Types of Adaptogen}

With a few exceptions, the chemical nature of the adaptogens has not yet been described in detail. Listed below are some features of a number of those that have been identified:

\section{(i) Saponins}

These are based on mevalonic acid. [21] They have been used traditionally as a general mental and physical tonic, providing medicinal benefit. They protect and restore homeostasis in the body, especially when under unremitting stress. It seems that the underlying mechanism of action of these has much in common with that of steroid hormones. Ginseng is a widely used member of this group. They are used to improve cognitive function, treat insomnia, and alleviate anxiety.

These agents also appear to possess anti-tumour and antimutagenic activity. Several individual ginsenosides suppress tumour cell growth, induce cell differentiation, regulate apoptosis and inhibit the formation of colonising groups of cancer cells, or metastases. Their mechanism of action apparently involves binding to the steroid molecules embedded in cancer cell membranes, so inhibiting their viability and frustrating cellular growth. [44]

Certain saponins may also exert their action by means of an anti-oxidant effect, although this is controversial at present. This mechanism of action is invoked to explain the anti-cancer and cardio-protective effects. In this connection, saponins also seem capable of promoting programmed cell death, or apoptosis, in cancer cells of patients with leukaemia.

\section{(ii) Triterpenes}

These also arise from the mevalonate metabolic pathway. [22] Amongst the products are, as well as the saponins, the phytosterols (such as beta-sitosterol), and the phytoecdysteroids (such as turkesterone).

\section{(iii) Oxylipins}

This group is a component of the acetate metabolic pathway, and comprises long-chain fatty acids (such as octadecadienoic acid) and a number of hydroxylated versions.

\section{(iv) Phenylpropanes}

The shikimate metabolic pathway is the source of these adaptogens. Typical products are the bio-flavonoids (such as the glucopyranosides) and the lignans (such as sesamin).

\subsection{Immune Modulators}

\subsubsection{Mechanism of Action}

These botanicals alter some activities of the immune system via the dynamic regulation of informational molecules - cytokines, hormones, neurotransmitters, and other messenger peptides. [23, 24] Many of the effects of herbal products are expressed by one or more groups of the cytokines (literally cell movers).

The cytokines are signalling molecules. They are small protein (peptide) or glycoprotein molecules released from a number of different cells in both the immune system and the nervous system, and carry regulating and mobilising signals between different cells within the body. There is a large, diverse group of these regulators, differing structurally, and with different functions. They include the interferons, the chemokines, the tumour necrosis factors (TNFs), and the interleukins. [25]

Cytokines maintain communication between organ systems, providing molecular cues for conserving physiological stability, that is, homeostasis. They orchestrate major sections of the immune response to infections and cancer. The onset and progress of many disorders, from cardiovascular disease to frailty, are thought to be influenced by cytokines. Although the cytokines were considered initially to act primarily as antiviral or anti-cancer agents, they are now regarded as crucial to inflammatory responses, cell growth, repair, and death.

Their role in the body is to assist in the removal of foreign bodies - viruses, bacteria, fungi, and even cellular debris. They instruct effector cells, such as the neutrophils, macrophages and monocytes to divide and mount an attack on these foreign bodies and digest the resulting remnants.

\subsubsection{Tolerance}

Most of the time, the immune modulators are effective in keeping the body's tissues free from the effects of microbe invasion. Sometimes, though, certain organisms can provoke 
a 'cytokine storm', which can have deleterious consequences. [26] Cytokine storms affect the young and healthy, who have well-developed immune systems, not the old or very young so much. The cytokines have been linked to a number of chronic diseases, including cancer, Alzheimer's disease, and clinical depression.

Severe reactions to, and even fatalities from, virus diseases such as SARS and influenza are the result of a cytokine storm, where a flood of cytokine molecules is released in response to the infection. This occurs for some reason, not yet understood. This leads to massive reactions from the body's organs and tissues, followed by collapse of internal metabolic processes, and sometimes death. The response to the infection is out of all proportion to the risk posed, so the cells which should be protecting the body actually cause it possibly serious harm.

\subsubsection{Clinical Application}

The interconnectedness of the body's multiple organ systems is well illustrated by disorders arising from inflammation of the cardiovascular system. As well as white blood cells, the liver, heart, vessel walls, and fatty tissues are known to produce cytokines. Conceivably any of these organs and tissues may contribute to inflammatory cardiovascular diseases, supporting the multifactorial nature, rather than single cause, of the onset of many diseases.

\subsubsection{Specific Herbs with Immune Modulatory Activity}

Several herbs have an effect on the secretion of cytokines from the immune cells. [27] In particular the secretion of cytokines interleukin- 6 and TNF-alpha, which are hostile to the body, is markedly reduced.

\section{(i) Garlic}

Garlic possesses the ability to modulate cytokine activity and increase the number of natural killer cells. It has shown an effect, albeit in an in vitro model, on a number of cytokines, including interleukins $-1,-6$ and -8 , and also TNF. Interleukin-1 is a pro-inflammatory cytokine, implicated in pancreatic beta-cell destruction and resulting hyperglycaemia. Garlic has also shown in animal models to stimulate secretion of interleukin-10, which is an antagonist of pro-inflammatory cytokines. A corresponding effect of garlic in humans has yet to be shown. These preliminary results provide some biochemical basis for the use of garlic in such diverse fields as diabetes, inflammatory bowel disease, and Alzheimer's disease.

Other herbal products where the putative mechanism of action has been identified are:

\section{(ii) St John's Wort}

This commonly used herb contains hypericin, and has been used for a long time to treat infection and reduce inflammation. It exhibits antiviral and anti-bacterial activity in vitro. In stimulating the immune system it most likely acts to some extent by reducing secretion of the two cytokines mentioned above. As a mood elevating herb, the antidepressant effect may be mediated by modulation of brain monoamine neurotransmitters, such as serotonin and noradrenaline.

\section{(iii) Turmeric}

This increasingly popular herb contains the polyphenol curcumin and has been shown to be a potent immunemodulator. Curcumin has well-defined modulating and antiinflammatory effects, and is able to prevent immune cells from responding to stimulants. The activation of the body's immune cells $-\mathrm{T}$ cells, B cells, neutrophils, macrophages, and natural killer cells - are modulated. Curcumin can also influence cytokine expression. This is achieved by downregulating various pro-inflammatory cytokines (TNF, interleukins and certain chemokines), possibly by inactivation of the transcription factor NF-kappa B. In addition, curcumin's modulatory potential is shown by its ability to enhance antibody response.

\section{(iv) Green Tea}

This herbal beverage contains high levels of catechin and other anti-oxidants, and is used as a tonic for the immune system. It has been shown to improve lymphocyte responses. In doing so it protects against cancer development. It also demonstrates some anti-viral activity in vitro.

\section{(v) Cat's Claw}

This South American herb is used to treat influenza sufferers. It contains a unique group of substances, the oxindole alkaloids, which are capable of stimulating the immune system. This acts by decreasing the production of cytokine TNF-alpha. These alkaloids, research suggests, help enhance white blood cell activity. Increasing the person's white blood cell count helps fight off microbial infections. Cat's claw is often used to treat inflammatory disorders. It contains substances which are remarkably potent inhibitors of TNF-alpha production. The main mechanism for its antiinflammatory activity, therefore, seems to be immunemodulation via suppression of TNF-alpha synthesis.

\subsection{Alkaloids}

More than 10,000 different alkaloids have been discovered, in over 300 plant families. [28] The biological roles of alkaloids have yet to be identified convincingly, in spite of extensive research over decades. The highly complex chemical structures rule out their being waste products or the final product in nitrogen metabolism, as much metabolic energy is expended in their biosynthesis.

It now appears that most alkaloids are part of the plants' defence and protection mechanisms. In the natural state, plants need protection from a variety of predators, from micro-organisms such as fungi, bacteria, and viruses, to parasites, insects, and grazing animals. Some alkaloids may be part of as yet undiscovered communication systems; neurotransmitters in the human (serotonin, dopamine, histamine) are similar structurally to certain plant alkaloids. Finally, alkaloids, nicotine especially, may be involved in cellular growth in parts of the plant, such as the root tips.

Many plants have been used medicinally purely on their 
alkaloid content, and these are still used in traditional and complementary medicine. For a hundred years or so ago, several alkaloids have been isolated from the parent plant, purified and incorporated into the pharmacopeia of conventional medicine. A number of these are shown in Table 1 (below):

Table 1. Alkaloids used historically, and clinical actions now used in modern medicine.

\begin{tabular}{llll}
\hline Plant & Alkaloid & Pharmacological response & Clinical application \\
\hline Belladonna & Atropine; scopolamine & Anti-cholinergic agent & Parkinson's disease Gut spasms; eye surgery \\
Periwinkle & Vinblastine; vincristine & Anti-tumour agent & Anti-cancer therapy \\
Poppy flower & Codeine & Anti-tussive agent & Cough suppressant \\
Rauwolfia & Reserpine & Anti-hypertensive agent & Lowers elevated blood pressure \\
Crocus & Colchicine & Anti-inflammatory agent & Relieves gout flare-ups Anti-cancer activity \\
\hline
\end{tabular}

A number of alkaloids have been used extensively for decades, and for a wide range of clinical conditions and procedures. Table 2 (below) lists a selection of these, plus their known mechanisms of action.

Table 2. Alkaloids used clinically in modern medicine, and their mechanism of action.

\begin{tabular}{|c|c|c|c|}
\hline Alkaloid & Source & Pharmacological action & Desired activity \\
\hline Atropine & Deadly nightshade & $\begin{array}{l}\text { Cholinergic receptor antagonist; anti- } \\
\text { muscarinic agent }\end{array}$ & $\begin{array}{l}\text { Low heart rate and heart block; pupil dilation in eye surgery; } \\
\text { antidote for some poisons; }\end{array}$ \\
\hline Reserpine & Indian snakeroot & Vesicular monoamine transporter inhibitor & Anti-psychotic; anti-hypertensive; \\
\hline Ephedrine & Ephedra & Sympathomimetic amine & Stimulant; decongestant; appetite suppressant \\
\hline Theophylline & Tea bush & $\begin{array}{l}\text { Adenosine receptor agonist; phosphodiesterase } \\
\text { inhibitor }\end{array}$ & $\begin{array}{l}\text { Bronchodilator in asthma; heart stimulant; nervous system } \\
\text { stimulant }\end{array}$ \\
\hline $\begin{array}{l}\text { Vinblastine } \\
\text { Vincristine }\end{array}$ & $\begin{array}{l}\text { Madagascar } \\
\text { periwinkle }\end{array}$ & Mitotic inhibitor; cytotoxic agent & Anti-cancer agent \\
\hline Caffeine & Coffee bush & $\begin{array}{l}\text { Adenosine receptor agonist; phosphodiesterase } \\
\text { inhibitor; glycine receptor antagonist }\end{array}$ & Nerve stimulant; exercise recovery; hair growth stimulant \\
\hline Paclitaxel & Pacific yew & Mitotic inhibitor & Anti-cancer; Kaposi sarcoma \\
\hline Noscapine & Poppy & Sigma receptor agonist & Cough suppressant; possibly anti-cancer activity \\
\hline Colchicine & Autumn crocus & Mitotic inhibitor & Anti-gout, anti-inflammatory agent; (poss.) anti-cancer activity \\
\hline Morphine & Poppy & Opioid receptor antagonist & Relief from moderate-severe pain \\
\hline
\end{tabular}

Many herbs in the natural state contain one or more alkaloids. Alkaloids are quite capable of provoking adverse drug reactions. [4, 29] Their use in high concentrations (which occur especially in liquid formulations), often causes diarrhoea, vomiting, and headache in sensitive individuals.

\subsection{Anti-inflammatory Agents}

Inflammation is part of a complex biological process which aims to restore homeostasis to the body after it has been disturbed by physical damage, chemical irritants, pathogenic micro-organisms, or damaged tissues. [30] It is arguably the body's major protective system, and works closely with the immune system in curbing pathological activity and initiating the healing process. It is modulated by interacting with both the endocrine and the nervous systems.

Conventional drug treatment involves the use of nonsteroidal anti-inflammatory drugs (NSAIDs) and corticosteroids. The former group acts by counteracting two enzymes, cyclo-oxygenase (which results in pain relief) and prostaglandin synthetase (which is involved in the signs and symptoms of inflammation). Corticosteroids work by attaching to steroid receptors in the cell's cytoplasm, then stimulating the nucleic acids.

The long-term use of NSAIDs can lead to erosions of the stomach lining, which often develop into serious and even life-threatening stomach ulcers and perforations. The risk of such adverse drug reactions increases in the elderly, the age group frequently affected by inflammatory disorders such as arthritis. The NSAIDs can also increase the risk of kidney damage and even asthmatic attacks.

Inflammation is part of the normal healing process, so there is no advantage to be gained clinically by completely suppressing this beneficial phenomenon. The total shut down of a healing process is often the outcome of conventional NSAIDs and exogenous corticosteroids, with the aim of suppressing symptoms of inflammation. What the person suffering from a persistent or severe inflammatory reaction needs is gentle control and regulation of the process. Several herbs are able to provide this without the adverse drug reactions which usually accompany conventional antiinflammatory agents.

Ginger, hyssop, arnica and turmeric are known to contain the inflammatory agent helenalin, Another anti-inflammatory agent, salicylic acid, is present in many herbs, including willow bark. Cannabinoids are being increasingly used effectively to treat intractable pain and unresponsive inflammation. [31]

\subsection{Nitric Oxide Synthesis Modulators}

\subsubsection{Background}

Nitric oxide (NO) is a highly reactive free-radical involved in muscle action and immune system functions. [33] It is a key messenger substance which plays an important role in several biochemical processes. It is also involved in a number of diseases, especially of the heart and blood circulation. [34] Chemically it is a simple gaseous compound, originally called the endothelial derived relaxing factor. It arises in the 
body from the metabolic reaction of oxygen with the amino acid arginine, catalysed by the enzyme nitric oxide synthase. NO can also result from the inorganic substance nitrate, which is present in the diet, or from breakdown of nitroglycerine, which is often given to treat angina pectoris. The site of synthesis is mainly the cells lining the blood vessels, or endothelium. Some is also produced in nerve cells, or neurons, where it serves as a neurotransmitter. In other tissues it can appear as a result of cytokine activity. As it is quickly broken down in seconds in the body, it is synthesised on site, according to local need. There is now substantial evidence that numerous single natural products and plant extracts can influence the production of NO in blood vessels.

\subsubsection{Role in the Body}

Nitric oxide diffuses freely across cell membranes, and acts to relax smooth muscle in blood vessels. The result is vasodilatation, that is, increased blood flow to the tissues serviced by the blood vessels. NO is therefore crucial to the cardiovascular system, because it assists the flow of blood through the heart and circulatory system. It also helps to remove dangerous blood clots, lower high blood pressure and inhibit plaque build-up in the arteries. A constant level of NO formation is important in protecting organs such as the liver from poor blood circulation, so reducing the risk of ischaemic damage. [35]

Generally, nitric oxide modulates homeostasis in blood vessels. It inhibits several adverse processes in the body which may develop into a pathological condition. The contraction of vascular smooth muscle is restrained, the unregulated growth and development of blood vessels is prevented, platelet clumping is inhibited, and the attachment of white blood cells to the inner endothelial lining of blood vessels is reduced. NO is also involved in inflammation, one of the body's main defence mechanisms for dealing with infection.

\subsubsection{NO in the Disease Process}

Reduced availability of NO in blood vessels encourages the onset and progress of cardiovascular disease. People with hypertension, diabetes, or atherosclerosis often show impaired NO synthesis, release or use. If its production is not tightly regulated, toxic damage to affected tissue occurs. At normal levels, NO dilates the blood vessels and so maintains satisfactory blood circulation. At low levels, blood circulation becomes sluggish, and this can give rise to certain disorders, such as angina pectoris and hypertension. Conversely, at abnormally high levels it causes circulatory shock, and may even induce tissue death. So diseases can develop both at very low levels, and at very high levels, of NO. Several disorders are known to be linked to wide swings in blood flow, such as arthritis and ulcerative colitis, juvenile diabetes, multiple sclerosis, and some cancers. [36]

The emergency vasodilators amyl nitrite and nitroglycerin are used in angina pectoris. They are converted to nitric oxide in the body. Sildenafil (aka Viagra) stimulates erections primarily by enhancing signaling through the nitric oxide pathway in the penile blood vessels.

\subsubsection{Diet and Nitric Oxide}

A number of everyday dietary components are known to activate or modulate the enzyme nitric oxide synthase. These include cocoa (active agent, epicatechin), black tea and green tea (flavanoids), pomegranates (polyphenols), olive oil (oleic acid and polyphenols), soy (genistein), and red wine (resveratrol). High levels of NO synthase activation encountered in vegetables and fruit, due to the presence of flavonoids, catechins, tannins and other polyphenolic compounds, are believed to contribute to their proven beneficial health effects. Vitamin $\mathrm{C}$ is thought to increase NO synthesis, so foods rich in this vitamin should have a beneficial effect on the blood circulation.

\subsubsection{Herbs and Nitric Oxide}

Several herbs increase NO production, and these are often used to treat cardiovascular disorders. One response is the activation of macrophages. These scavenger cells, located in the various tissues, engulf and digest invading microbes. Other herbs act as vasodilators by increasing NO in vascular tissue. For example, the saponins from ginseng (specifically, the ginsenosides), are known to relax blood vessels. This probably contributes to the fatigue-relieving and blood pressure-lowering effects of ginseng. In addition, herbal products from ginkgo and hawthorn have been shown to affect endothelial NO production.

Safed musli, a herb used as a traditional tonic, improves reproductive function in animals by stimulating NO synthesis. Gingko biloba acts via platelet-activating factor (PAF) which is a mediator of inflammation, several leucocyte functions, platelet activity, and directly implicated in asthma and other allergies. It is also involved in the anaphylactic response. PAF acts directly on vascular smooth muscle by stimulating endothelium-derived growth factor and prostacyclin, which leads to smooth muscle relaxation. The net effect is dilated arteries and arterioles to and within the lung, as well as the rest of the body, increasing blood flow, and better oxygen and glucose supply to tissues. G. biloba also inhibits the enzymes which are involved in smooth muscle relaxation, and is said to be effective in relieving the bronchiolar constriction associated with asthma and other allergy-related respiratory diseases.

\subsection{Micro-nutrients}

A micro-nutrient, aka trace element, is the general term which includes micro-minerals, vitamins, essential fatty acids, and amino acids. [37] Their role in the body is not providing energy or building tissues, but assisting existing enzyme systems operate more efficiently. Micro-minerals comprise the metallic elements iron, chromium, zinc, copper, cobalt, manganese, and molybdenum.

Micro-nutrients also protect against environmental toxins which are capable of damaging the body, especially if exposed to them over time. If an essential nutrient is missing from the diet, or in short supply, or lacking the potency to 
keep the natural balance within the body, the body systems become distressed. This has a 'knock-on' effect on other organs, so that the body as a whole becomes unbalanced. This, for example, is what happens when a person is deficient in a certain nutrient, or malnourished in some way. [38]

Herbal products contain a host of nutritional factors minerals (such as potassium), vitamins (especially the watersoluble B complex), and trace elements (molybdenum, zinc, cobalt). These may contribute to resolving chronic deficiency disorders in the patient. These may be minor in terms of symptoms, but they may have a disproportionate impact on the person's well-being.

As mentioned (above) the mechanism of action of many micro-nutrients is to act as co-factors for certain enzymes. Zinc, for example, is essential for activity of several enzymes, including alcohol dehydrogenase, phosphodiesterase, and aminopeptidase. Iron is not only an important co-factor for several enzymes involved in energy production in the mitochondria, but also is an essential component of non-enzyme proteins such as haemaglobin and cytochrome.

Many herbal remedies contain micro-minerals, and these may contribute to the tonic activity claimed. This response does of course depend on the type and amount of the micromineral, and on the underlying deficiency manifesting in the patient.

Vitamins are often present in particular herbal products, albeit at low levels. Vitamin $\mathrm{C}$, found in several products, assists in the absorption of dietary iron, so reducing the risk of iron-deficiency anaemia. Conversely, the herbal product's components may assist in the absorption of specific vitamins provided by the diet, or from vitamin supplements. Two flavonoid glycosides, rutin and hesperidin, which are present in some herbal products, help in the absorption of Vitamin C from the digestive tract.

\section{Discussion}

There is a revival in interest for herbal remedies in many parts of the developed world. [3,40] They are increasingly being used these days as therapeutic agents for many chronic disorders, or combined with minerals and vitamins in health enhancing supplements, or as invigorating tonics and teas.[9] This has arisen for several real and important reasons. One of these is increasing awareness of the negative aspects of some conventional drugs, particularly adverse drug reactions, both in the short and long term, and suspicions about the real extent to which they occur. This trend also reflects the urge towards a more natural lifestyle, and a new-found respect for natural or holistic healing. Yet another is the instinctive need to deal with the underlying causes of a particular ailment, rather than merely suppressing the troubling symptoms. Herbal remedies often claim to do this, in contrast to conventional medicine, which appears to be more concerned with symptom suppression and restoration of normal laboratory parameters. [7]

However, there is an understandable reluctance on the part of physicians who are science and evidence based to use herbal remedies, for several important reasons. One is the lack of accurate, objective and reproducible information regarding pharmacological action. [39, 40] Practitioners of modern conventional medicine really need to be convinced that they work. There is a large degree of doubt about this, ranging from scepticism to total rejection. These realistic attitudes can, however, be assuaged by incontrovertible evidence of herbal efficacy. Not only will this make them more relaxed and inclined to use herbal remedies where they feel they are justified, but they will consider then seriously as partner to conventional drugs in integrative medicine. They will also be provided with information which helps them avoid herb-drug interactions. [41, 42]

Other measures for greater acceptance of herbal remedies are, of course, necessary. One is satisfactory proof of genuine identity of the product is essential: developments in DNA analysis of botanicals can help here. Another is the impact of the use of herbal remedies on plant ecology: protection of sensitive or vulnerable plant species demands better control, and more intensive controlled cultivation. Yet another is the unavoidable need for quality control procedures to ensure that the patient, as consumer, is receiving the correct remedy, at the stated dosage, within the laid-down time limits.

Research into the modes of action of common herbal remedies is therefore an important prerequisite for broad acceptance of herbal remedies into general medical practice. We are aware that very few actual plant species have been investigated for therapeutic potential, compared to the vast number that presently exist on earth. This should serve as a major incentive for further research.

\section{Conclusion}

Over the last decade or so there has been a rapid increase in the body of technical literature on the mechanism of action of a large number of herbal remedies. This new-found knowledge has been generated by credible investigators, not only in academia and state-run institutions, but also in a number of pharmaceutical companies. The basis for effective clinical action of many of these has been vindicated, and their broad usage confidently adopted. Many other herbal remedies have, however, been proven less effective than claimed by their adherents, and their usage decisively rejected and abandoned. There are three good reasons for the search for proven pharmacological action. First, the use of herbal remedies, either alone or combined with other treatment modalities, needs to be based on a solid understanding of their pharmacology. If this is not available, the credibility of herbal treatment generally suffers, and its true value diminished. Second, the search requires the standardisation of the herbal product in terms of unambiguous identity of herbal components, efficacy per unit weight, formulation into standard doses, and its chemical stability. Third, if and when herbal remedies are considered as part of integrative medicine, their modes of action need to be understood. To date, a number of discreet mechanisms of 
action of a wide range of commonly used herbal products have been confirmed. They range from adaptogenic properties, immune modulation and nitric oxide synthesis modulators, to micro-nutrient and anti-inflammatory activity. For the future, other pharmacological actions will be attributed to other herbs. This increased knowledge will boost the confidence of healthcare practitioners in their use of herbal products, alone or together as part of Integrative Medicine.

\section{Acknowledgements}

The authors, Prof Rashid Bhikha and Dr John P Glynn, would like to thank Ms Magdalene du Sart for her unstinting and uncomplaining support in the preparation of this publication. It is greatly appreciated.

\section{References}

[1] Schaffner KF. Assessments of efficacy in biomedicine: the turn toward methodological pluralism. In: Callahan D, ed. The role of complementary and alternative medicine: accommodating pluralism. Washington, DC: Georgetown University Press; 2002. p. 7.

[2] Tilburt JC and Kaptchuk TJ. (2008) Bulletin of the World Health Organization Vol 86:594-599.

[3] Glynn JP and Bhikha RA. (2017) The pharmacology of Western drugs. Asian Journal of Science and Technology. Vol 8, No 7 (5) 100-5106.

[4] The Pharmacological Basis of Therapeutics. Goodman and Gilman ( $9^{\text {th }}$ Ed.) (1996). McGraw-Hill, USA

[5] Thomas, P. (2002). What Works What Doesn't - The Guide to Alternative Healthcare. Gill \& Macmillan Ltd, Dublin.

[6] Miller FG, Emanuel EJ, Rosenstein DL, Straus SE. Ethical issues concerning research in complementary and alternative medicine. JAMA 2004; 291:599-604.

[7] Ernst, Edzard. Shedding the light. Department of Experimental Pharmacology, University of Naples, 17.08.2004. Available online at: www.theguardian.com/lifeandstyle/2004/aug/17/healthandwel lbeing.health

[8] Firenzuolini and Gori L. Herbal Medicine Today: Clinical and Research Issues. Evidence Based Complement Alternative Medicine (2007) Sept 4(Suppl 1):37-40.

[9] Van der Merwe, A. Herbal Remedies (2002) pp 35-44. Tafelberg (Cape Town).

[10] Herbal Medicine, Biomolecular and Clinical Aspects. Eds: Benzie IFF and Wachtel-Galor S (2011) (2nd edition) CRS Press/Taylor \& Francis.

[11] Page SW and Maddison JE. Principles of Clinical pharmacology (2008). 2nd Edition Elsevier DV.

[12] Wink M. Modes of Action of Herbal Medicines and Plant Secondary Metabolites. Medicines (Basel). (2015) Sep; 2(3): 251-286.
[13] Weiss, R, and Fintelmann, V. Herbal Medicine (2000). G T Verlag, Stuttgart, Germany.

[14] Bhikha, R. A. H. and Haq, M. A. (2000). Tibb: Traditional Roots of Medicine in Modern Routes to Health. Mountain of Light. South Africa.

[15] Brekhman, I. I. and Dardymov, I. V. (1969). "New Substances of Plant Origin which Increase Nonspecific Resistance". Annual Review of Pharmacology Vol 9: 419-430.

[16] Panossian A and Wagner H. Stimulatory effects of Adaptogens: Am overview with particular reference to their efficacy following single-dose administration (2005). Phytotherapy Research Vol 19, 819-838

[17] Ray A, Gulati K, Anand R (2016) Stress, Adaptogens and Their Evaluation: An Overview. J Pharma Reports Vol 1:110.

[18] Panossian A. Understanding adaptogenic activity: specificity of the pharmacological action of adaptogens and other phytochemicals. (2017) Annals of the New York Academy of sciences. Aug;140(1):49-64.

[19] Chen T-S, Liou S-Y and Chang Y-L. (2008). Antioxidant Evaluation of Three Adaptogen Extracts. Amer. J Chinese Medicine. Vol 36, No 06.

[20] Ajala, Tosin O. The Effects of Adaptogens on the Physical and Psychological Symptoms of Chronic Stress (2017). Georgia State Honors College Undergraduate Research Journal: Vol. 4, Art 2.

[21] Botanical Medicine in Clinical Practice (2008). Ed: Watson RR and Preedy VR. CAB Intern., UK.

[22] Triterpenes - Advances in Research and Application. (2013) Ed: Ashton Acton Q. Scholarly Edition, Georgia, USA.

[23] Spelman, $\mathrm{K}$ et al. Modulation of cytokine expression by traditional medicines: A review of herbal immuno-modulators. Alt Med Rev. (2006) Vol 11 (2); 128-32.

[24] Belardelli F and Ferrantini M. (2002) Cytokines as a link between innate and adaptive antitumor immunity. Trends Immunol; Vol 23:201-208.

[25] Ho RJY and Gibaldi M. (2013). Biotechnology and Biopharmaceuticals: Transforming Proteins and Genes into Drugs. John Wiley \& Sons Inc, USA.

[26] Canna S and Behrens EM. (2012). Making Sense of the Cytokine Storm: a conceptual framework for understanding, diagnosing and treating hemophagocytic syndromes. Pediatric Clinical North America:Vol 59 (2):329-344.

[27] Barak V, Birkenfeld S, Halperin T and Kalickman I (2002). The effect of herbal remedies on the production of human inflammatory and anti-inflammatory cytokines. Isr Med Assoc J. Vol 4 (11 Suppl):919-22.

[28] Babbar N. (2015) An introduction to alkaloids and their applications in pharmaceutical chemistry The Pharma Innovation Journal Vol 4(10): 74-75.

[29] Saxena M, Saxena J, Nema R, Singand D and Gupta A. (2013) Phytochemistry of Medicinal Plants, Journal of Pharmacognosy and Phytochemistry Vol.1 (6):168-172.

[30] Wink M. (2014) Alkaloids: Toxicology and Health Effects. Available online at: www.researchgate.net/publication/301702655_Alkaloids_Toxi cology_and_Health_Effects 
[31] Apurba Sarker Apu, Shakhawat Hossan Bhuyan, et al (2012). Anti-inflammatory activity of medicinal plants native to Bangladesh: A review. Journal of Applied Pharmaceutical Science Vol 2 (22):07-10.

[32] Richardson JD, Kilo S and Hargreaves KM. C (1998). Cannabinoids reduce hyperalgesia and inflammation via interaction with peripheral CB1 receptors. Pain Vol 75:111119 .

[33] The Burning Question About Inflammation: Are Cannabinoids the Cure? (2017). The Scientist. Available online at: www.the-scientist.com/sponsored-multimedia/theburning-question-about-inflammation-are-cannabinoids-thecure-31134.

[34] Kuo-Hsiung Lee, Morris-Natdchke, Quian K et al (2012). Recent Progress of Research on Herbal Products Used in Traditional Chinese Medicine. J. Tradit. Complement. Med. 2012 an-Mar; 2(1): 6-26.

[35] Mukherjee P, Cimelli MA, Kang S and Silverman RB. (2014). Development Of Nitric Oxide Synthase Inhibitors for Neurodegeneration and Neuropathic Pain Chemical Society Reviews Vol 43 (19) 6814-6838.

[36] Rosselli M, Keller PJ and Dubey RK (1998). Role of nitric oxide in the biology, physiology and pathophysiology of reproduction. Human Reproduction Update Jan-Feb; Vol $4(1): 3-24$.
[37] Ciery c and Zipp C. (2011). Nitric oxide in health and disease: its role in the practice of medicine. Osteopathic Family Physician Vol 3(2) 66-73.

[38] Kumar, A, Singh, R. and Singh, N. (2011) Analysis of macro and micro nutrients in some Indian medicinal herbs grown in Jaunpur (u.p.) soil. Natural Science Vol 3, 551-555.

[39] 8. Evans, S. Changing the knowledge base in Western herbal medicine (2008). Social Science and Medicine. Vol 67(12) 2098-2106.

[40] Ekor, M. (2013). The growing use of herbal medicines: issues relating to adverse reactions and challenges in monitoring safety. Front Pharmacol. 4: 177.

[41] McCabe BJ. (2004). Prevention of food-drug interactions with special emphasis on older adults. Curr. Opin. Clin. Nutr. \& Metabol. Care. 7(1); 21-26.

[42] Hu Z, Yang X, Ho PC, Chan SY, Heng PW, Chan E, Duan W, Koh HL, Zhou S. (2005). Herb-drug interactions: A literature review. Drugs. 65(9):1239-82.

[43] Lucas R. Natures Medicines. The folklore, romance and value of herbal remedies (1968). Neville Spearman, London, UK.

[44] Leung KW and Wong AS-T (2010). Pharmacology of ginsenosides: a literature review. Chinese Medicine Vol 5; 20. 